\title{
Un regard en arrière du rédacteur sortant de charge
}

\author{
Th. Abelin'
}

En tant que rédacteur sortant de charge, c'est volontiers que j'accepte l'invitation de mes successeurs de procéder à une brève rétrospective des huit ans et demi pendant lesquels m'a été confieé la responsabilité du contenu de Médecine sociale et préventive et de sa présentation. Ce fut une période marquante de développement de la médecine sociale et préventive dans le monde et de définition plus précise du profil de cette discipline en Suisse. Notre revue s'est donné la tâche de participer activement, pas à pas, à ces développements et d'en rapprocher ses lecteurs. Si elle y est parvenue, et si elle est parvenue aussi en traitant de préoccupations actuelles à renforcer elle-même certaines évolutions désirables, elle a alors atteint le but qu'elle s'était fixé.

Un survol des 26 cahiers à thème qui sont parus depuis mi-1972 permet de faire défiler devant nous, une fois encore, quelques tendances des années 1970 dans le domaine de la santé publique et de la médecine sociale et préventive. Le premier numéro à thème de cette série déjà $\left(\mathrm{N}^{\circ} 1-2 / 1973\right)$ rappelle ce qui est probablement l'événement le plus important de cette décennie du point de vue de l'histoire de la médecine et de la santé, dans la mesure où il traitait de la vaccination antivariolique à la lumière du succès de la campagne d'éradication de ce fléau. Sept ans plus tard, en mai 1980, l'Organisation mondiale de la santé pouvait annoncer que, à l'échelon mondial, la variole était vaincue. Un chapitre de l'histoire de l'humanité et de ses souffrances était ainsi clos. Les deux numéros à thème suivants ( $\mathrm{N}^{\circ}$ 4/1973 et $\left.\mathrm{N}^{\circ} 1 / 1974\right)$, de même qu'un numéro ultérieur ( $\left.\mathrm{N}^{\circ} 1 / 1976\right)$, devaient s'attacher à d'autres épidémies affectant toute la planète, qui ne sont pour l'instant en aucune façon vaincues et dont l'OMS doit se préoccuper maintenant de façon croissante: il s'agit des problèmes de consommation de drogues, d'alcool et de tabac. De nombreux articles et éditoriaux ont été consacrés ces dernières années aux dépendances; ils reflètent l'importance que leur accorde la médecine sociale et préventive suisse. Des cahiers sur la prophylaxie des maladies dentaires $\left(\mathrm{N}^{\circ}\right.$ 6/1975), sur des questions d'actualité en hygiène hospitalière ( $\left.N^{\circ} 5-6 / 1974\right)$, sur la santé et le sport ( $\mathrm{N}^{\circ} 6 /$ 1976) et sur la prévention du suicide ( $\left.\mathrm{N}^{\circ} 1 / 1979\right)$ ont permis de résumer l'état des connaissances sur des aspects donnés de la prévention, alors que cinq cahiers étaient consacrés aux problèmes des modifications de l'environnement entraînées par une technicisation croissante et à leurs conséquences pour l'homme $\left(\mathrm{N}^{\text {os }}\right.$ $3 / 1974,2 / 1975,2-3 / 1976,3 / 1977$ et 6/1980). A deux reprises, un bilan a été dressé des développements conceptuels et méthodologiques dans des secteurs importants de la médecine préventive (Examens médicaux préventifs, $\mathrm{N}^{\circ} 3-4 / 1975$; Education pour la santé, $\mathrm{N}^{\circ} 5$ et $\left.6 / 1977\right)$. Et, à trois reprises, des questions médico-sociales et de pédiatrie sociale ont été au centre de numéros à thème: prise en charge d'enfants à risque, troubles du comportement chez l'enfant, enfants handicapés (Nos $2 / 1974,1-2 / 1977$ et 6 / 1979).

Plusieurs numéros traitant des aspects structurels et organisationnels de secteurs importants, publiés depuis 1978 , rendent compte d'une orientation actuelle de l'action de médecine sociale et préventive à l'échelon international: cela non seulement à propos de médecine préventive ( $\mathrm{N}^{\text {as }} 3 / 1978$ et 5-6/1978), mais encore de médecine du travail ( $\mathrm{N}^{\circ} 1 / 1978$ ), de la coopération au développement dans le domaine médicosanitaire $\left(\mathrm{N}^{\text {os }} 2-3 / 1979\right)$ et du système de santé en général $\left(\mathrm{N}^{\text {os }} 2 / 1978\right.$ et $\left.1-2 / 1980\right)$. Alors que, pendant les années 1950 et 1960, l'épidémiologie des maladies chroniques avait permis de découvrir de nombreux «points d'accrochage» pour la prévention, on a vu dès le début des années 1970 combien leur réalisation pratique était complexe, dans un système marqué surtout par la médecine curative et dans une société menée d'abord par des considérations économiques à court terme. L'analyse systématique dans le domaine de la santé publique (health services research) et l'évaluation systématique de mesures préventives novatrices, orientées vers la collectivité, furent introduites et trouvèrent leur traduction en Suisse dans

'Prof. Dr med., directeur de l'Institut de médecine sociale et préventive de l'Université de Berne, Hôpital de l'Ile, 3010 Berne. 
deux Programmes nationaux de recherche (PNR), qui influenceront de façon importante, dans les années 1980, les développements du système de santé et de la médecine sociale et préventive. Dans un des derniers cahiers parus durant mon mandat de rédacteur $\left(\mathrm{N}^{\circ} 5 / 1980\right)$ ont pu être présentés les premiers résultats du Programme national de recherche 1A (Prophylaxie des maladies cardio-vasculaires), cependant que le premier numéro publié sous l'égide de la nouvelle Rédaction ( $\mathrm{N}^{0}$ 1-2/1981) était consacré au PNR 8 (Economie et efficacité du système suisse de santé). Ces deux cahiers ne témoignent pas seulement du mouvement vers une recherche sur les services de santé toujours plus systématique, orientée vers la pratique et interdisciplinaire, cadre dans lequel le spécialiste formé de médecine sociale et préventive doit jouer un rôle important de coordination; ils reflètent aussi la collaboration étroite et amicale qui caractérise la médecine sociale et préventive de notre pays. C'est grâce à cet excellent esprit et à la disponibilité et à l'engagement des rédacteurs responsables de numéros à thème et des auteurs que la tâche de rédacteur de Médecine sociale et préventive fut, constamment, un plaisir pour moi. Au chapitre des remerciements, il convient de mentionner particulièrement ici les secrétaires de rédaction avec lesquelles j'ai eu la chance de travailler, Mmes Thérèse Dethurens, Verena Krattiger, Amélie Bürgi, Maria Wagner et Ursula Bucher, de même que les collaborateurs des Editions Orell-Füssli SA (jusqu'en 1976) et Vogt-Schild SA (depuis 1977), qui, «dans les coulisses», ont fait en sorte que les manuscrits confiés à la Rédaction soient, à chaque fois, imprimés à temps et sous une forme attrayante. Il faut remercier aussi les deux rédacteurs francophones de cette période, Olivier Jeanneret (jusqu'en 1977) et Jean Martin (depuis 1978), grâce auxquels les auteurs et lecteurs francophones ont trouvé leur dû linguistique dans les pages de notre revue. A eux tous vont aujourd'hui mes vifs remerciements, de même qu'à mes successeurs Fred Epstein, Rudolf Bruppacher et Jean Martin, qui ont repris, sans aucune friction, la conduite de la Rédaction. Je leur souhaite pour les années à venir une activité aussi stimulante et satisfaisante que celle que j'ai eu le privilège d'avoir. 